\title{
Fading testbed for free-space optical communications
}

\author{
Amita Shrestha, Dirk Giggenbach, Ahmad Mustafa, Jorge Pacheco-Labrador, Julio Ramirez, Fabian \\ Rein
}

German Aerospace Center (DLR), Institute of Communications and Navigation, 82234 Wessling

\begin{abstract}
Free-space optical (FSO) communication is a very attractive technology offering very high throughput without spectral regulation constraints, yet allowing small antennas (telescopes) and tap-proof communication. However, the transmitted signal has to travel through the atmosphere where it gets influenced by atmospheric turbulence, causing scintillation of the received signal. In addition, climatic effects like fogs, clouds and rain also affect the signal significantly. Moreover, FSO being a line of sight communication requires precise pointing and tracking of the telescopes, which otherwise also causes fading. To achieve error-free transmission, various mitigation techniques like aperture averaging, adaptive optics, transmitter diversity, sophisticated coding and modulation schemes are being investigated and implemented. Evaluating the performance of such systems under controlled conditions is very difficult in field trials since the atmospheric situation constantly changes, and the target scenario (e.g. on aircraft or satellites) is not easily accessible for test purposes. Therefore, with the motivation to be able to test and verify a system under laboratory conditions, DLR has developed a fading testbed that can emulate most realistic channel conditions. The main principle of the fading testbed is to control the input current of a variable optical attenuator such that it attenuates the incoming signal according to the loaded power vector. The sampling frequency and mean power of the vector can be optionally changed according to requirements. This paper provides a brief introduction to software and hardware development of the fading testbed and measurement results showing its accuracy and application scenarios.
\end{abstract}

Keywords: FSO, Optical Communication, Laser Communication, Fading emulator, testbed

\section{INTRODUCTION}

Free-space optical (FSO) communication offers wide and free spectrum for wireless communication to meet the increasing demand of high data rate. However, FSO is challenging because of different atmospheric effects that deteriorate the incoming signal. In addition, FSO being a line-of-sight communication, inaccurate pointing and tracking also causes intermediate signal reduction. Fading occurs when the received power is less than certain threshold, below which the signal cannot be correctly sampled, and scintillation is the measure of the fluctuation of received power. In order to cope with such challenges, researchers are investigating different mitigation techniques like sophisticated coding and interleaving, aperture averaging by using bigger telescopes, transmitter diversity by using more than one transmitters, adaptive optics, etc. After theoretical investigation and simulation, selected solutions are implemented and tested repeatedly in laboratory, various field tests should be done to fully verify the system in realistic channel for every changes made in the system. This is very time consuming and often specific environmental conditions cannot be repeated at arbitrary times. With the motivation to avoid time consuming field trials, the Optical Communications Group in DLR has been developing a system that can emulate the realistic fading channel using measured data. In addition, the group is very active in scientific demonstration of different missions and research ${ }^{1}$ and therefore has numerous power vectors taken during aircraft downlinks, satellite downlinks, and ground to ground links. The measured power vectors are normalized and saved in a database. These vectors are called power vector (PV) or fading vector in rest of the paper.

Various versions of fading testbed (FTB) were developed within DLR. A first version was invented in $2010^{2}$, using rotating transparent plates with varying transmissivity patterns, which attenuated a passing laser beam and thus created fading. The timely behavior could be influenced by the rotation speed, and the strength of fading by the patterns mounted onto the wheels. Later, a fiber based fading testbed was developed using the variable optical attenuator $\left(\mathrm{VOA}^{3}\right)$ and a combined sampling and analog-signal generator device ("Handyscope" ${ }^{4}$ ). The basic principle of the testbed is to drive 
the VOA by voltage that can be controlled using the Handyscope. However, the performance of the VOA was not as good as expected from the datasheet, since the VOA should have been current driven, but was voltage driven in this first setup. Therefore, for later versions, a current driver circuit was implemented which could convert the voltage-output from the Handyscope to required current for driving the $\mathrm{VOA}^{5}$. Open-loop control software was used to steer the VOAcurrent to attenuate the optical signal, but the resulting optical fading was far from emulating the real optical fading channel. Another version of the fading testbed comprised an additional optical attenuator to increase the dynamic range of the input and a feedback photodetector to enable a closed loop control of the VOA-current.

Meanwhile, with similar motivation, diverse testbeds and simulation software versions were developed by various organizations. For example scintillation playback system using optical modulator by Moore et.al. ${ }^{6}$, turbulence generator by Hippler et. al. ${ }^{7}$, fading simulator for satellite-to-ground optical communication by Takashi ${ }^{8}$ et. al., etc. The main focus of our testbed is to regenerate recorded power vector in the laboratory and emulate the intensity effect of the channel to the signal. This allows improving systems for certain scenarios and evaluating in the same environment without need to repeat the experiment in the field. However, it does not include effects of the optical phase

\section{FADING TESTBED}

\subsection{Basic overview}

Block diagram with basic overview of the components used in fading testbed is shown in Figure 2-1 and briefly explained below.

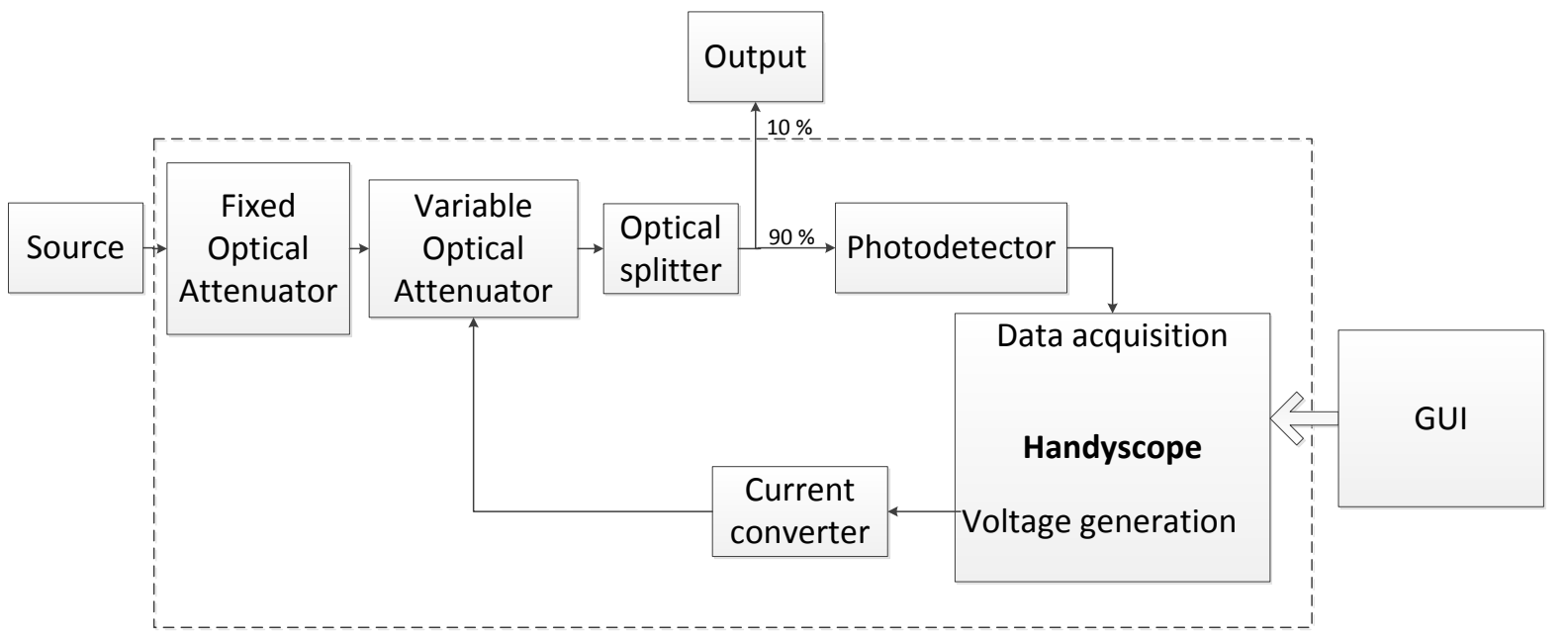

Figure 2-1. Basic block diagram of the fading testbed

1. Source: Input signal to the testbed is the optical signal that will be affected by emulated FSO channel inside the testbed. Optical fiber can be connected to input connector of the testbed.

2. Fixed optical attenuator ${ }^{9}$ : The input signal goes through the optical attenuator whose attenuation can be changed with the help of screw. It works as a manually controlled fixed attenuator for the testbed. It can be used to attenuate the incoming signal from the source to match the maximum acceptable power for the user.

3. Variable optical Attenuator (VOA): VOA is main part of the fading testbed. As name suggests, it attenuates the input signal according to its driving current. Therefore, the VOA driving circuit is developed that accepts the variable voltage coming from the signal generator and produces the driving current for VOA according to required fading vector.

4. Handyscope: It is used for data acquisition and voltage generation according to loaded power vector. This device include digital oscilloscope which reads output of the photodetector and a signal generator which generates voltages to drive the VOA via current driving circuit according to the loaded power vector. 
5. Current driving circuit for VOA: The circuit is built to drive and to electrically control the VOA. Using this circuit, user can apply a voltage from an arbitrary waveform generator to control the attenuation in a fiber system.

6. Optical splitter: Optical splitter is used to split the output signal of VOA such that some part of the signal can be used for feedback purpose and rest of the signal can be sent to the output. For the current setup of the testbed, $90 \%$ of the output is connected to the feedback photodetector and $10 \%$ goes to the output of the detector.

7. Photodetector: Photodetector is used for feedback purpose. One output of the optical splitter is connected to device under test (DUT) and another to the photodetector. It is mainly used in the calibration process to measure and calculate the output power for given input VOA voltage.

8. Graphical user interface: Graphical user interface is developed in C++ using Qt library. It allows user to load a power vector, select the required mean power of the output, calibrate the testbed, etc. The screenshot of the GUI showing different options are shown in Figure 2-5.

Front-view of the fading testbed in Figure 2-2 shows all interfaces and top-view of the testbed in Figure 2-3 shows how each components are connected. The specification of the fading testbed is summarized in Table 1.

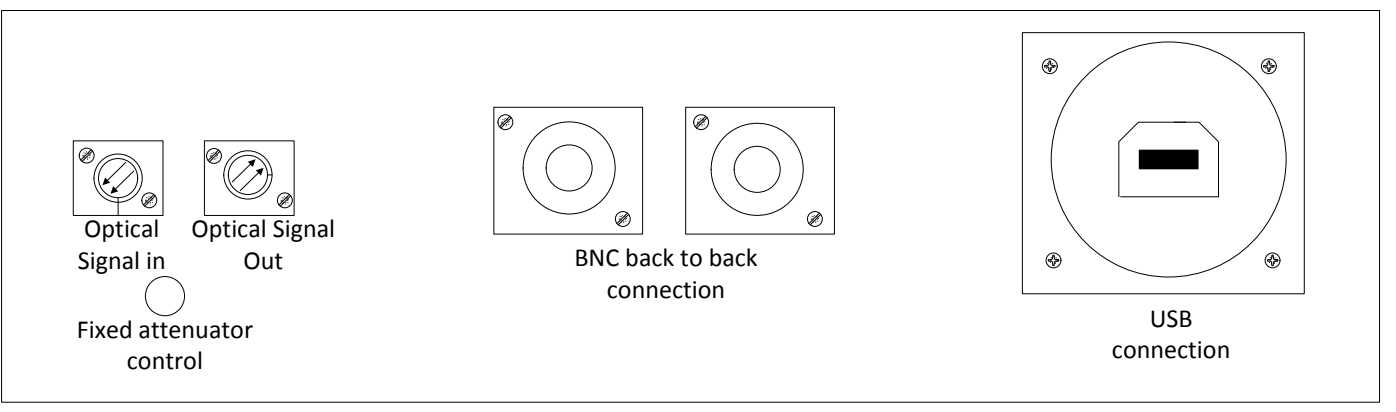

Figure 2-2: Front-view of the Fading Testbed

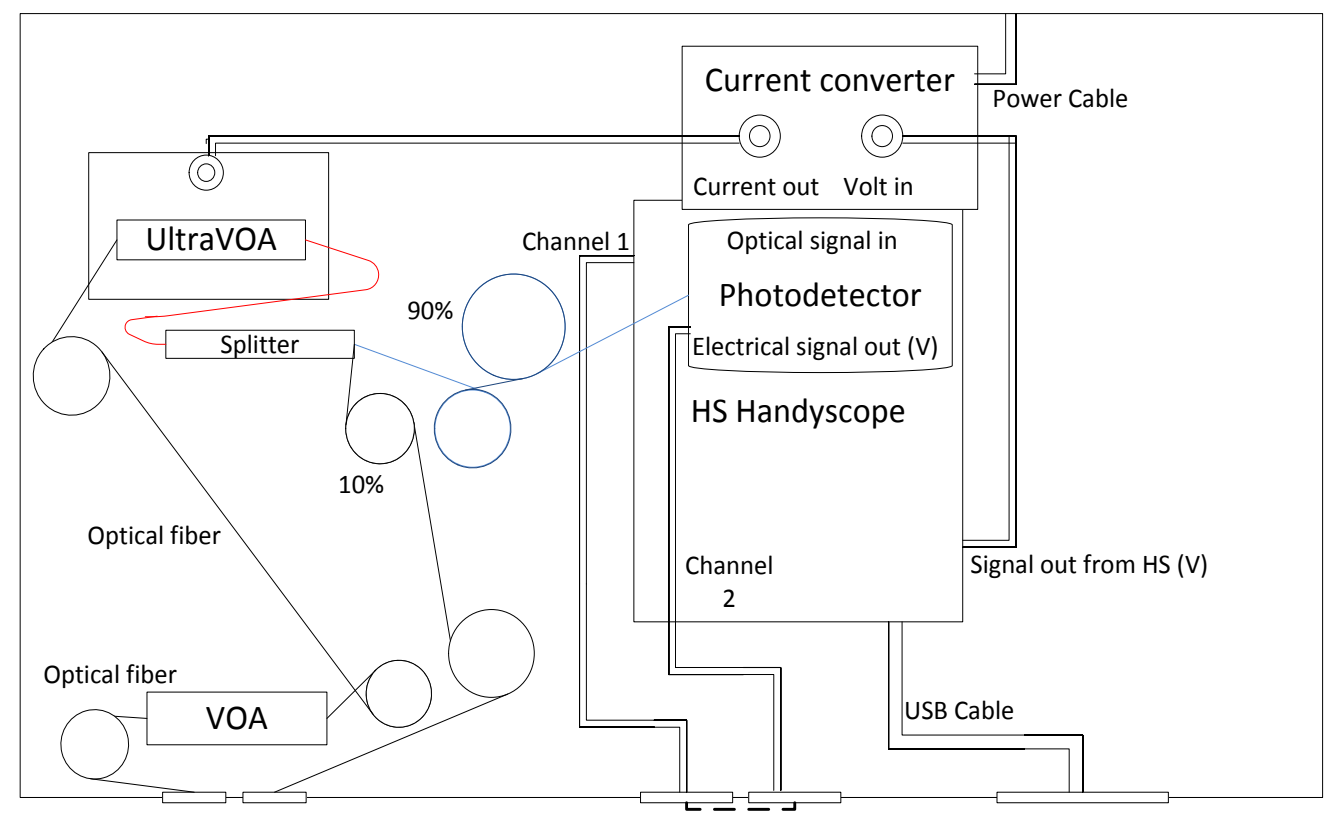

Figure 2-3: Top view of the Fading Testbed 
Table 1. Specification of the fading testbed

\begin{tabular}{|l|l|}
\hline \multicolumn{1}{|c|}{ Parameter } & \multicolumn{1}{c|}{ Values } \\
\hline Optical Wavelength & $1525-1568 \mathrm{~nm}$ \\
\hline Optical input power & $\leq 300 \mathrm{~mW}$ \\
\hline Minimum Response time & $1 \mu \mathrm{s}$ \\
\hline Operating temperature & $0-75^{\circ} \mathrm{C}$ \\
\hline Resolution of the Handyscope & $14 \mathrm{bit}$ \\
\hline Weight & $2.6 \mathrm{Kg}$ \\
\hline Dimension & $430 \times 225 \times 90 \mathrm{~mm}$ \\
\hline
\end{tabular}

\subsection{Working principle}

Control software for fading testbed is written in C++ and user interface is designed using Qt library. The user interface can be used to control and read the status of the testbed. Detailed flow chart of the software is shown in Figure 2-6. Working principle of fading testbed can be explained in mainly four steps:

1. Initialization: Initially the "Initialization” button shall be clicked to initialize the handyscope. If initialized successfully, it would display the initialization information and date in the Graphical User Interface (GUI).

2. Parameter Settings: Then different parameters like required mean power, power vector to be regenerated, sampling rate of the vector, etc. can be set from GUI.

3. Calibration: Once all parameters are entered, calibration is done. This is because there are various components in the testbed and each component has some losses and behavior uncertainty that can deviate results from expected. Therefore, calibration shall be done every time the testbed is used. During calibration, signal generator in Handyscope generates voltage in certain steps such that it drives VOA to produce different attenuation levels and corresponding power at the detector which is $90 \%$ or $10 \%$ of the total output produced by VOA, is measured and final output power of the testbed is calculated. The photodetector has certain offset which should be compensated. Therefore, prior to any calibration, photodetector offset is measured and given to the software. Power at the output $\left(\mathrm{P}_{\text {out }}(\mathrm{mw})\right)$ of the testbed is calculated as shown below.

$$
\mathrm{P}_{\text {out }}=\left(\left(\frac{V_{P D}-\text { Voffset }_{P D}}{R_{\text {load }} \times \mathrm{R}_{P D}}\right) \times \text { factor }\right)
$$

where,

$\mathrm{P}_{\text {out }}=$ output power of the testbed

$V_{P D}=$ voltage detected at feedback photodetector

Voffset $_{P D}=$ offset voltage at the photodetector

$R_{\text {load }}=$ load resistance conencted to the photodetector

$R_{P D}=$ Responsivity of the photodetector

factor $=$ factor of total power connected to the output

Finally, a calibration table is created listing values of voltage generated by signal generator and corresponding output power at DUT. The table is saved in an internal memory of the signal generator that can later be quickly fetched while regenerating a power vector. Figure 2-4 represents one of the calibration table and it does not include complete dynamics of the VOA because of inaccuracy of the feedback detector. For higher VOA voltages generating higher attenuation, the power detected by photodetector was noisy, thus causing inaccuracy in the calibration table which in turn would create inaccuracies in the regenerated power vector. This can be 
improved by replacing the detector by less noisy detector (PDA10CS ${ }^{10}$ ) with transimpedance amplifier. The detector has been identified and needs to be integrated in next version of the fading testbed.

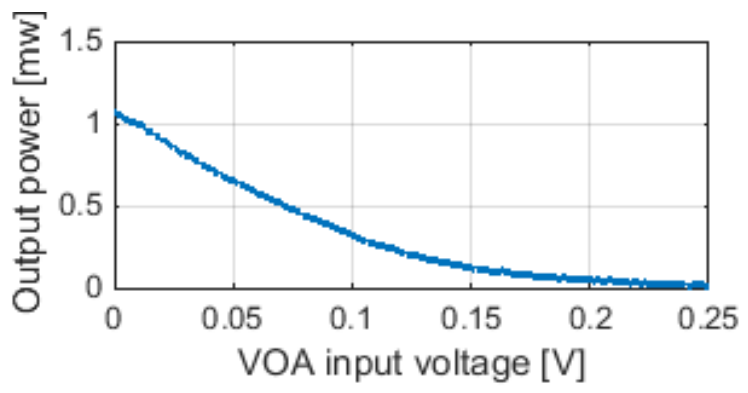

Figure 2-4. Calibration table example showing VOA input voltage required to produce corresponding power at the output

4. Power vector regeneration and measurement: After the calibration process is complete, either the loaded power vector with given mean power or fixed power equal to given mean power can be generated depending on the user input. It is important to note that the power vector has to be normalized before loading it to the testbed. The normalized power vector is then multiplied by the user-defined mean power to create the desired power vector. Now, software reads list of exact powers that have to be generated and fetches the corresponding required voltage from the calibration table. It saves the list of corresponding voltages to be generated in the internal memory of the handyscope. Then it starts generating the voltages at user-defined sampling rate, thereby producing the required instantaneous output power. Meanwhile, output power can be monitored by feedback photodetector and correction can be applied by adjusting voltage, if needed.

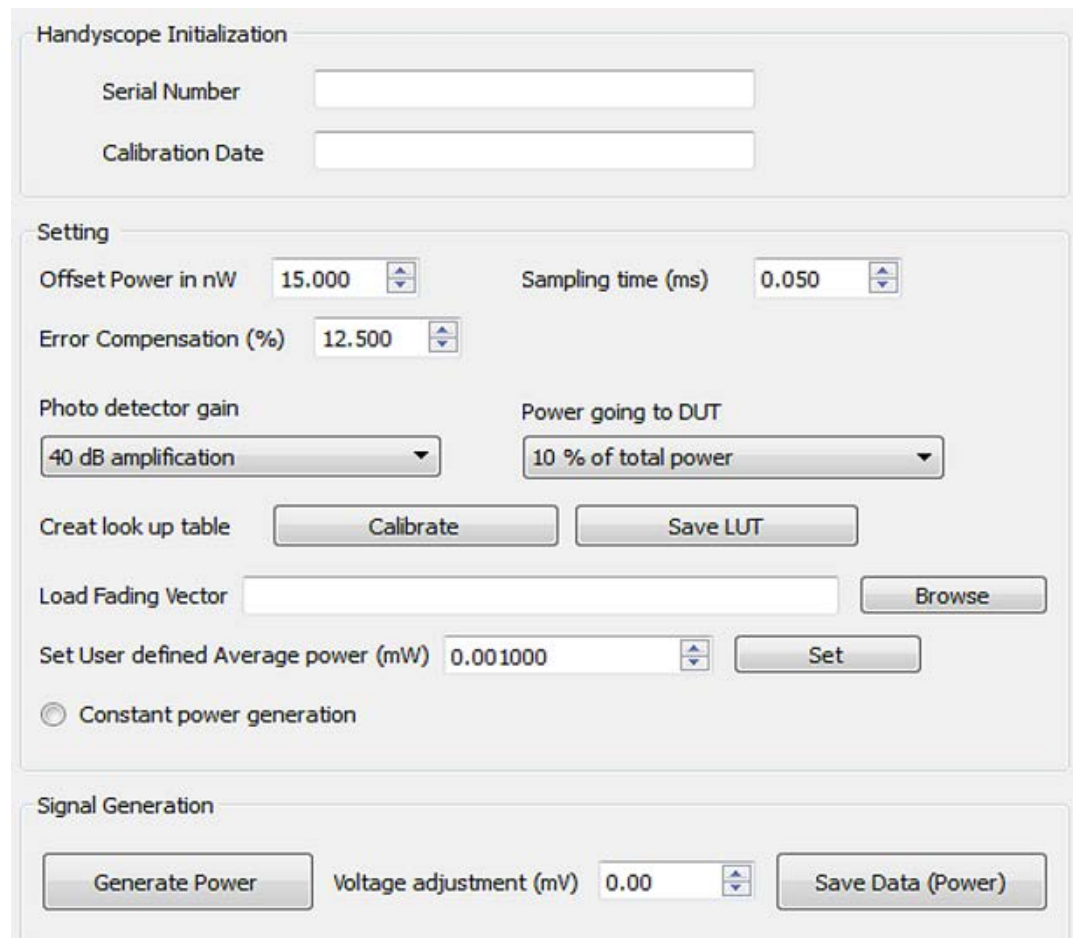

Figure 2-5. Graphical User Interface (GUI) for Fading Testbed 


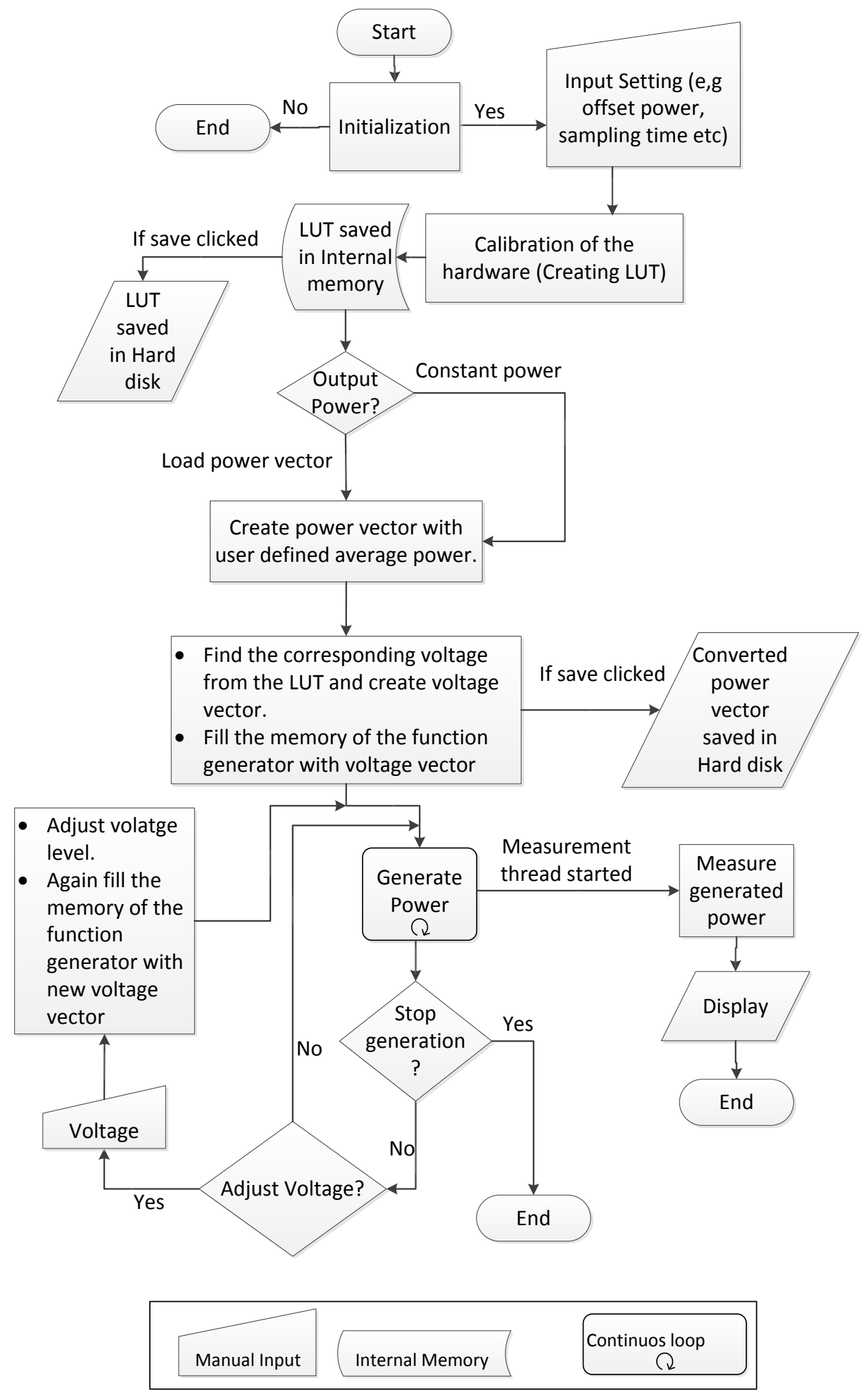

Figure 2-6: Flow chart of the Fading Testbed software 


\section{PERFORMANCE}

There are various important factors regenerating the power vector to emulate real channel condition, mainly timing and amplitude accuracy. In order to speed up the process of producing the required output power both converted power vector and calibration table is saved in an internal memory of the signal generator. Time response of VOA was measured by applying different driving current to VOA and minimum response time with good result was measured to be $5 \mu s^{5}$. In order to evaluate the performance of the testbed, a measured power vector from GEO uplink ArtemEx campaign ${ }^{11}$ was loaded and is compared with regenerated power vector. The picture of the setup is shown in Figure 3-1. Constant optical input signal was connected to the FTB and the output power was connected to the photodetector and photodetector voltage was recorded using 16-bit picoscope.

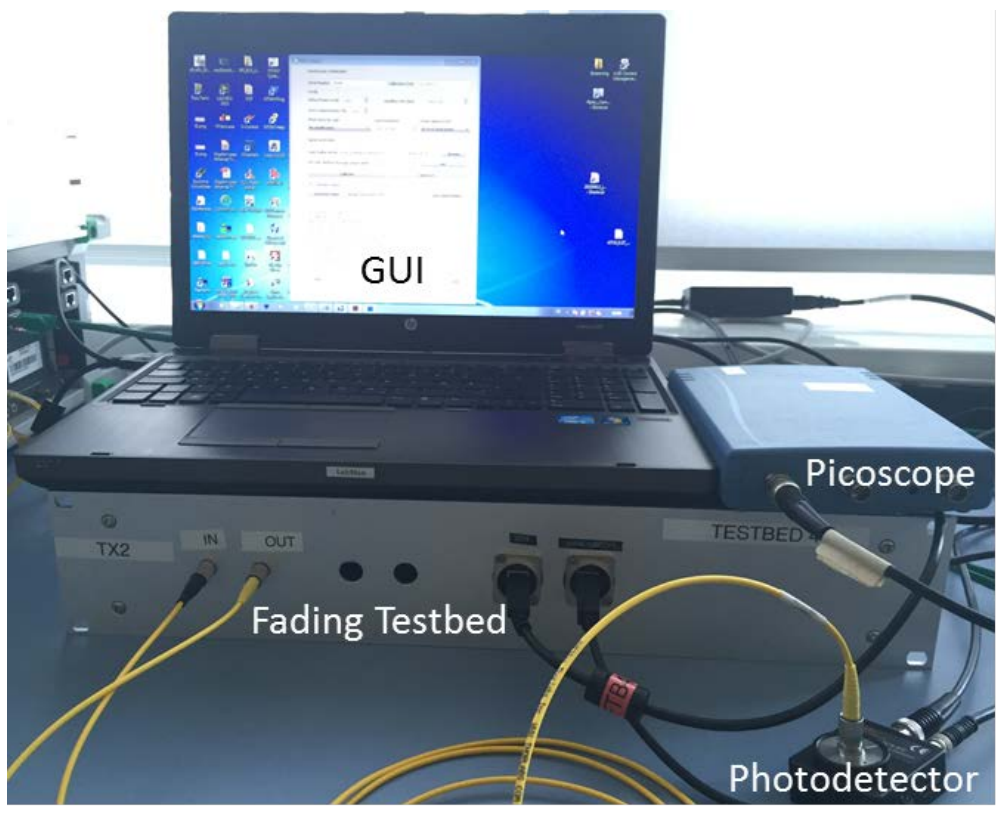

Figure 3-1. Setup for regenerating PV using Fading Testbed

First 200ms of vectors are presented in Figure 3-2. Cross correlation between original and regenerated power vectors is shown in Figure 3-3. Furthermore, Figure 3-4 shows how probability distribution function (PDF) of regenerated vector overlaps with PDF curve for original power vectors. Different parameters like mean power, standard deviation and Power Scintilaltion Index (PSI) of original and regenerated PV are summarized in Table 2. PSI is the measure of fluctuation of optical signal and is calculated as the ratio of variance of the signal and square of its mean.

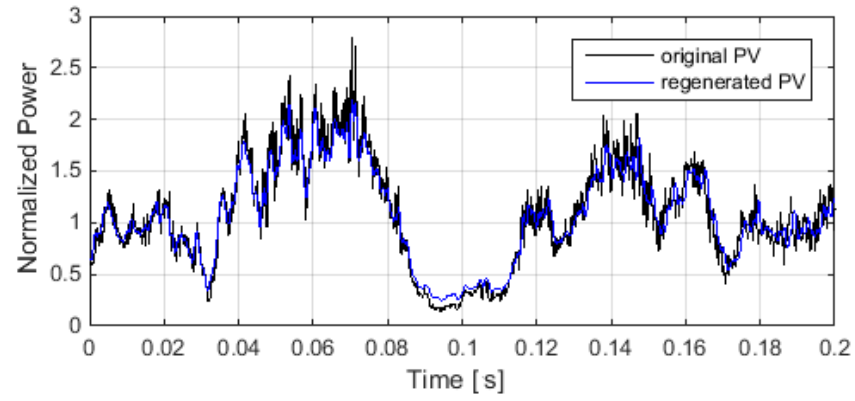

Figure 3-2.Original vs regenerated power vector (first 200ms of the complete vector

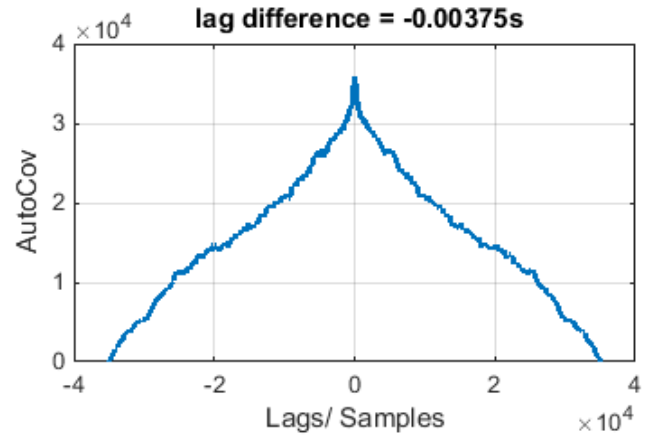

Figure 3-3. Cross correlation of orignal PV and regenerated PV 


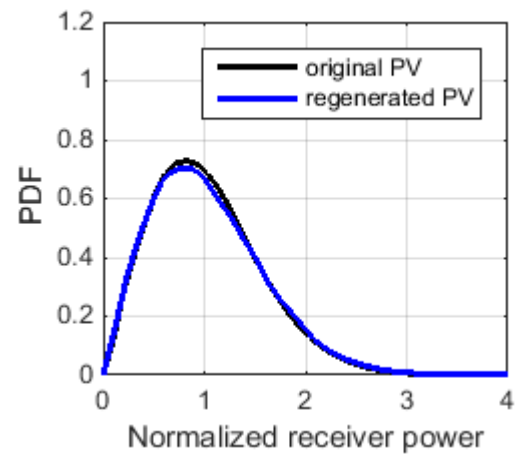

Table 2. Parameters for the real PV and the regenerated PV

\begin{tabular}{|l|l|l|}
\hline Parameter & Required & RG-PV \\
\hline mean & 0.9018 & 0.9065 \\
\hline $\begin{array}{l}\text { Standard } \\
\text { deviation }\end{array}$ & 0.4403 & 0.4412 \\
\hline PSI & 0.238 & 0.208 \\
\hline
\end{tabular}

Figure 3-4. PDF of original and regenerated PV

\section{APPLICATION}

The fading testbed has been very useful tool for development and evaluation of different components of FSO. Some examples of projects within DLR that are using fading testbed are mentioned below:

\subsection{Development of robust FSO Laser Ethernet Transceiver}

RF forward error correction (FEC) schemes alone are not robust enough to protect the data in a FSO channel. This is due to the nature of the errors which present a high correlation during periods of time in the range of $10 \mathrm{~ms}$. For this reason DLR is developing Laser Ethernet transceiver $\left(\mathrm{LET}^{12}\right)$ which is a media converter between Ethernet frames and proprietary LET frames for FSO. It features novel fading-robust communication protocols combined with error correction schemes to allow error free transmission through the atmosphere. The protection is done using two ReedSolomon codes (inner and outer code) in a product code configuration. Additionally, an interleaver is included for providing protection against long duration fades. In this regard, the use of the FTB provides a pseudo-controlled laboratory conditions to test and validate different novel FEC schemes for LET. The advantage of the FTB is that it can either provide a deterministic fading pattern or it can replicate a previously measured channel scenario. This second option is done by measuring the received power during a campaign, save it as a power vector and later replicates it with the FTB.
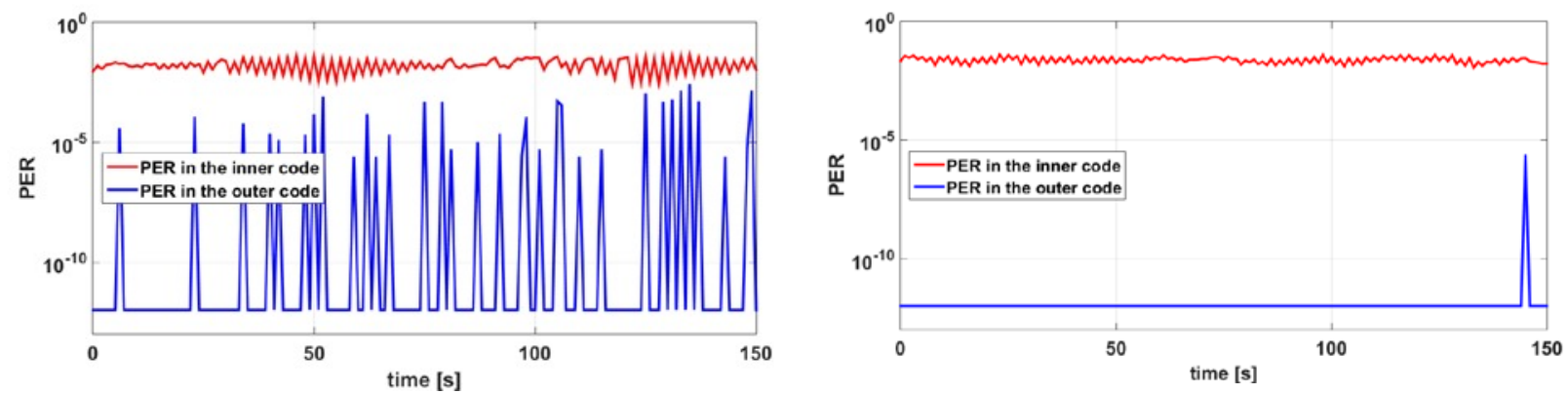

Figure 4-1. LET statistics without interleaver (left) and with interleaver (right)

In this example, a power vector of the ArtemEx campaign ${ }^{11}$ has been replicated with the FTB to analyze the performance of LET system. Figure 4-1presents the result of an experiment using the LET prototype with (right) and without (left) the interleaver. In both figures the red curve represents the packet error rate (PER) in the inner code, which is the one closest to the channel. The blue curve represents the PER in the outer code and when it achieves a value of $10^{-12}$ indicates that the data could be recovered completely. These results prove that the inclusion of an interleaver significantly improves the protection against long duration fades. Moreover, further studies can be done in order to optimize the product code structure and the deepness of the interleaver. 


\subsection{Transmitter Diversity application}

Transmitter diversity is a technique to mitigate the fading effects in the uplink channel. The benefits of transmitter diversity scheme are verified in the uplink for geostationary satellite in ArtemEx campaign ${ }^{13}$. The efficiency of the FTB is verified in a lab experiment on transmitter diversity where the testbed emulated the power vector measured during ArtemEx campaign ${ }^{11}$.

Figure 4-2 shows a realization of the transmitter diversity scheme. By placing two or more transmitter telescopes at moderate spatial separation on ground, the turbulent paths are de-correlated and the fading effects on the uplink signal are reduced using standard diversity schemes.

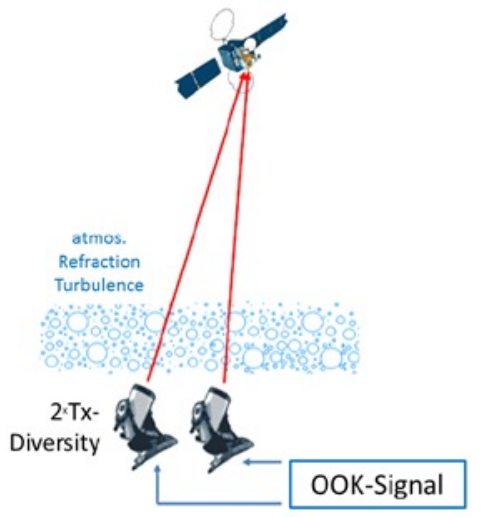

Figure 4-2. Spatial diversity scheme with two transmitters in a GEO feeder uplink

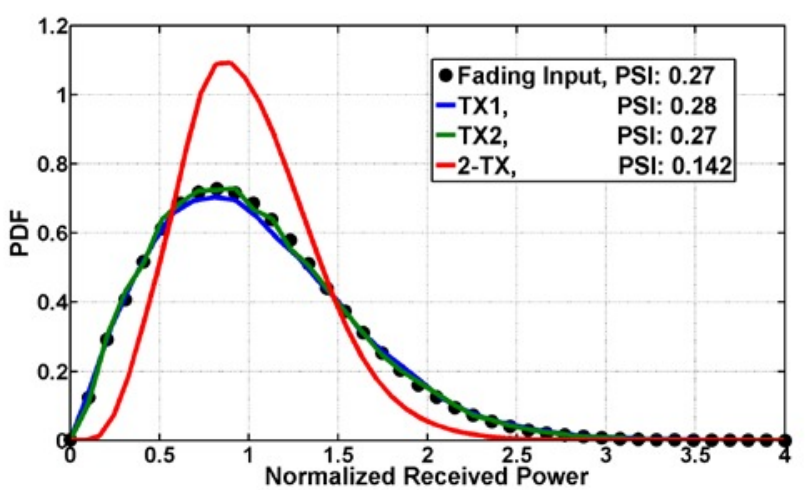

Figure 4-3. Probability density function obtained from the input and output power vectors using fading testbed

The two uncorrelated paths are emulated from two statistically independent fading testbeds which successfully emulated the scintillation effects of the turbulent atmosphere. The testbed is loaded with measured power vector from ArtemEx measurement campaign carried out for the uplink GEO feeder link. Both testbeds are loaded with the same fading vector but with a random non-zero time delay to make the output from the two testbeds completely uncorrelated as it is in the outdoor environment. Two optical signals experience the scintillation effects in the testbed. Measurements are repeated by switching on only one transmitter at a time and then switching on both to verify the improvement in signal stability due to diversity. Mean received power in all three cases is kept same to show that the improvement is due to reduced outage probability and not because of higher SNR when using two beams. In Figure 4-3, probability density distribution (PDF) of the received powers in the three cases is presented.

It can be seen that the received power distributions for transmitter 1(TX1) and transmitter 2 (TX2) match closely with the fading input given to the fading testbed verifying the ability of the testbed to regenerate the power vector with good accuracy. The result shows that the sum of the two beams reduces the variance, hence providing less fading strength compared to the case when only one transmitter is used to send the data signal in up-link. As next step, the testbed is planned to be used in a Terabit/s long range free space optical transmission experiment ${ }^{14}$. It will emulate the power vector measured between DLR site in Weilheim-Lichtenau and Hohenpeißenberg. Using this fading testbed, the performance of high throughput communication system dense wavelength division multiplexing (DWDM) technique can be optimized in laboratory before actually performing the experiment in the outdoor environment

\section{CONCLUSION}

Fading testbed using variable optical attenuator was developed that could emulate the real optical channel by regenerating measured power vectors. The testbed is still under development and various improvements like including more sensitive and less noisy feedback detector, automatic error compensation by using feedback detector readings are foreseen. Moreover, an additional VOA is planned to be installed to increase overall dynamics of the system. FTB has been widely used in different projects within DLR and is being very useful tool for testing different systems including coding schemes for FSO transceivers, tracking stability of different FSO terminals, transmitter diversity system, etc. 


\section{REFERENCES}

[1] D. Giggenbach, F. Moll, and N. Perlot, “Optical Communication Experiments at DLR,” Journal of the National Institute of Information and Communications Technology, vol. 59, 2012.

[2] D. Giggenbach and M. Brechtelsbauer, "Daempfungsscheiben-Fadingsimulator fuer atmosphaerisches Labortestbed [Fadingsimulator mit Teufelsraedern],”, October 2010.

[3] Kotura, UltraVOATM Single-Channel, 2011.

[4] Handyscope HS3 Instrument manual Rev. 2.2.

[5] K. Shahiduzzaman, "Development and testing of the Testbench for Optical communication transceivers," 2013.

[6] C.I. Moore, M.F. Stell, W.S. Rabinovich, H.R. Burris, M.R. Suite, L.W. Thomas, and R. Mahon, "Naval Research Laboratory scintillation playback system for bench top lasercomm testing," Atmospheric Propagation VII, L.M. Wasiczko Thomas and E.J.E. Spillar, eds., SPIE-Intl Soc Optical Eng, 2010.

[7] S. Hippler, T. Henning, F. Hormuth, D. Butler, and W. Brandner, "Generation of atmosphere-like optical turbulence,” SPIE-The international Society for Optical Engineering, 2006.

[8] S. Takashi, T. Morio, and T. Hideki, "Fading Simulator for Satellite-to-Ground Optical Communication,” 2012.

[9] Single Mode, Variable Fiber Optical Attenuators, www.thorlabs.com: Thorlabs, .

[10] PDA10CS InGaAs Switchable Gain Detector User Guide.

[11] A. Mustafa, D. Giggenbach, J. Poliak, A. Shrestha, R. Mata-Calvo, and C. Fuchs, "Lab Implementation of 10Gbps/channel Optical Transmitter Diversity Scheme for Geostationary Satellite Feeder Links,” 16th ITG Symposium on Photonic Networks, 2015.

[12] J. Ramirez, A. Shrestha, S. Parthasarathi, and D. Giggenbach, "Gigabit Laser Ethernet Transceiver for Free-Space Optical Communication Systems,” Application of Lasers for Sensing \& Free Space Communication (LS\&C), IEEE, 2013.

[13] R.M. Calvo, P. Becker, D. Giggenbach, F. Moll, M. Schwarzer, M. Hinz, and Z. Sodnik, "Transmitter diversity verification on ARTEMIS geostationary satellite,” SPIE Photonics West, 2014.

[14] D. Giggenbach, J. Poliak, R. Mata-Calvo, C. Fuchs, N. Perlot, R. Freund, and T. Richter, "Preliminary Results of Terabit-per-second Long-Range Free-Space Optical Transmission Experiment THRUST,” Proc. of SPIE, 2015. 